\title{
Clinical guidelines on the application of Internet of Things (IOT) medical technology in the rehabilitation of chronic obstructive pulmonary disease
}

\author{
Guiling Xiang ${ }^{1 \#}$, Xiaodan Zhu ${ }^{1 \#}$, Lin $\mathrm{Ma}^{2 \#}$, Huai Huang ${ }^{1 \#}$, Xiaodan $\mathrm{Wu}^{1}$, Wei Zhang ${ }^{3}$, Shanqun $\mathrm{Li}^{1}$; \\ on behalf of the Writing Expert Group of Expert Consensus on Clinical Application of IOT Medical \\ Technology in the Rehabilitation of Chronic Obstructive Pulmonary Disease, the Respiratory Disease \\ Rehabilitation Professional Committee of China Medical Education Association* \\ ${ }^{1}$ Department of Pulmonary Medicine, Zhongshan Hospital, Fudan University, Shanghai, China; ${ }^{2}$ Department of Pulmonary Medicine, the First \\ Affiliated Hospital of Nanchang University, Nanchang, China; ${ }^{3}$ Department of Pulmonary Medicine, Shuguang Hospital Affiliated to Shanghai \\ University of Traditional Chinese Medicine, Shanghai, China \\ \#These authors contributed equally to this work. \\ Correspondence to: Dr. Shanqun Li; Dr. Xiaodan Wu. Department of Pulmonary Medicine, Zhongshan Hospital, Fudan University, 180 Fenglin \\ Road, Xuhui District, Shanghai 200032, China. Email: li.shanqun@zs-hospital.sh.cn; wu.xiaodan@zs-hospital.sh.cn. Dr. Wei Zhang. Department of \\ Pulmonary Medicine, Shuguang Hospital Affiliated to Shanghai University of Traditional Chinese Medicine, 528 Zhangheng Road, Pudong New \\ Area, Shanghai 201203, China. Email: zhangw1190@sina.com.
}

Submitted Apr 15, 2021. Accepted for publication Jun 22, 2021.

doi: $10.21037 /$ jtd-21-670

View this article at: https://dx.doi.org/10.21037/jtd-21-670

\section{Introduction}

Chronic obstructive pulmonary disease (COPD) is one of the most common preventable and treatable chronic respiratory diseases, characterized by persistent respiratory symptoms and incomplete reversible airflow limitation caused by external toxic and harmful gases or particulate materials, with progressive decline in lung function, and a series of clinical manifestations of systemic co-morbidities

\footnotetext{
* Members of the Writing Expert Group of Expert Consensus on Clinical Application of IOT Medical Technology in the Rehabilitation of Chronic Obstructive Pulmonary Disease, the Respiratory Disease Rehabilitation Professional Committee of China Medical Education Association(listed in alphabetical order), is shown below:

1. Guangxi Li, Department of Pulmonary Medicine, Guang'anmen Hospital, Chinese Academy of Chinese Medical Sciences, Beijing 100053, China. 2. Guiling Xiang, Shanqun Li, Xiaodan Wu, Zilong Liu, Xiaodan Zhu, Department of Pulmonary Medicine, Zhongshan Hospital, Fudan University, Shanghai 200032, China. 3. Kaifeng Xu, Department of Respiratory and Critical Care Medicine, Peking Union Medical College Hospital, Chinese Academy of Chinese Medical Sciences, Beijing 100730, China. 4. Jin Yan, Department of Nursing, the Third Xiangya Hospital of Central South University, Changsha 410205, China. 5. Linfu Zhou, Department of Respiratory and Critical Care Medicine, the First Affiliated Hospital of Nanjing Medical University, Nanjing 210029, China. 6. Liyan Jiang, Department of Pulmonary Medicine, Shanghai Chest Hospital, Shanghai Jiao Tong University, Shanghai 200433, China. 7. Lin Ma, Wei Zhang, Department of Pulmonary Medicine, the First Affiliated Hospital of Nanchang University, Nanchang 330006, China. 8. Wen Li, Department of Respiratory Medicine, Second Affiliated Hospital of Zhejiang University, Zhejiang University, Hangzhou 310009, China. 9. Qing Miao, Department of Pulmonary Medicine, Beijing Xiyuan Hospital, Chinese Academy of Chinese Medical Sciences, Beijing 100091, China. 10. Wei Wang, Department of Pulmonary Medicine, the First Affiliated Hospital of China Medical University, Shenyang 110001, China. 11. Wei Zhang, Department of Pulmonary Medicine, Shuguang Hospital Affiliated to Shanghai University of Traditional Chinese Medicine, Shanghai 201203, China. 12. Ziwen Zhao, Department of Pulmonary Medicine, Guangzhou First People's Hospital, Guangzhou 510013, China.
} 
such as abnormal skeletal muscle function and mental depression during the long-term course of the disease, in which the limited exercise tolerance seriously affects the life quality of patients. COPD is a major cause of chronic morbidity and mortality worldwide. Existing studies showed that in 2012, 3 million patients worldwide died from COPD, accounting for about $6 \%$ of the total causes of death. In China, the prevalence of COPD accounts for $8.2 \%$ of the prevalence of people over 40 years of age, and the number of patients is about 100 million $(1,2)$.

Pulmonary rehabilitation (PR) was defined by European Respiratory Society (ERS) and American Thoracic Society (ATS) in 2013: PR is a multidisciplinary and comprehensive intervention for promoting healthy behaviors that can be sustained over time, through exercise training, education and behavior change, following the principle of individualized patient treatment, to improve the behavioral and psychological status of patients with chronic respiratory diseases (3). ERS and ATS proposed in 2017 that PR is indicated for the overwhelming majority of patients with COPD, including those with hypercapnia (4).

The Global initiative for Chronic Obstructive Lung Disease (GOLD) 2019 defined a standard PR program as aerobic exercise and resistance or strength training for at least 30 minutes twice a week for 6 to 8 weeks, which could improve dyspnea and health status, and improve exercise tolerance in patients with COPD (5). PR has been shown to enhance exercise tolerance, improve life quality, relieve dyspnea and reduce readmission rates in patients with COPD, etc. Its effectiveness in improving lung function has been demonstrated. PR programs could be implemented for a patient with chronic airway disease who has longterm respiratory symptoms, limited activity, or who has not achieved a satisfactory treatment outcome despite standardized pharmacological therapies, especially for those with different severity of COPD. PR has become an integral part of the treatment of a patient in the stable phase of COPD, and GOLD has made pulmonary rehabilitation, especially lower extremity exercise and respiratory muscle training, the core and cornerstone of $\mathrm{PR}$ programs for patients with COPD (6). However, PR for patients with COPD has been promoted earlier in foreign countries, but is still in the initial stage in China. The majority of hospitals do not really carry out pulmonary rehabilitation, which is mainly promoted in some first-tier developed cities, while rare in hospitals and primary hospitals in second or thirdtier cities. At present, for patients with COPD, the PR is still limited to exercise assessment and training, treatment is valued over prevention, and the health literacy is low. Patients have no concept of health management, do not receive regular treatment, lack of a standardized out-ofhospital rehabilitation management system, and cannot adhere to rehabilitation treatment after discharge.

In addition, PR for patients with COPD faces many challenges and has the following problems: the large number of patients who need to receive rehabilitation, the lack of professional rehabilitation health care professionals, the lack of professional institutions and professionals with a high level of standardized COPD rehabilitation, the large gaps in medical resources and physician experience between hospitals of different grades and between hospitals in large cities and remote areas, low technical mastery and low acceptance by medical staff of COPD rehabilitation in remote areas and primary hospitals. At the same time, the large hospitals are overcrowded with rehabilitated patients and are unable to meet the clinical needs of patients. In addition, there are many practical problems such as limited mobility due to the symptoms, transportation inconvenience, old age and frailty, low education level, and poor compliance. All of these ultimately lead to low willingness to undergo pulmonary rehabilitation and low compliance in patients with COPD. Therefore, how to improve the clinical medical and management level of pulmonary rehabilitation and enhance the quality of pulmonary rehabilitation as soon as possible has become an urgent issue in the pulmonary rehabilitation program for patients with COPD.

The Internet of Things (IOT) is the fourth technological revolution in the global information industry after computers, the Internet and mobile communication networks (7). It connects various information sensing devices such as radio frequency identification (RFID) devices, infrared sensors and global positioning systems through the Internet, thus forming a huge network. The core of IOT is an extended and expanded network based on the Internet. The user side can be extended and expanded to interchange and communicate information between any object and thing (8). The team of Professors Chunxue Bai and Shanqun Li from the Department of Pulmonary Medicine, Zhongshan Hospital, Fudan University, creatively applied IOT medical technology to the management of chronic respiratory diseases, pointing out that this new diagnosis and treatment model can turn patients from passive treatment to early warning and active treatment, greatly reducing the cost of diagnosis and treatment work, as well as the problem of scarce medical resources in less 
developed areas (9). Mengsun Yu, academician of Chinese Academy of Engineering, proposed the concept of "Health Internet of Things", pointing out that the mainstream direction of future medical development is a new Internet of Things health management model that can manage human health (including disease) in a network. With these two concepts, the research of IOT in medical field has been raised to a macro level (10). The emergence of IOT medicine provides a new technological platform to solve the above dilemma. In the field of rehabilitation medicine, IOT has been applied to remote physical therapy, exoskeleton medical robots, cognitive impairment, etc. It was emphasized in GOLD2020 that PR in the community or at home with network support was no less effective than face-to-face PR in a hospital with medical device assistance (6).

Compared with traditional pulmonary rehabilitation, the advantages of applying IOT medical technology to manage patients with COPD to complete PR are as follows: (I) improve compliance: by creating a platform for communication and feedback between doctors, nurses and patients, providing patients with humanized spiritual support and medical services, and facilitating the exchange of information between health care professionals and patients and between patients, so that patients can regain confidence and actively cooperate with pulmonary rehabilitation; (II) amplify the effect of famous doctors: it is convenient for doctors who carry out PR to manage more patients, so that patients from all over the country can get their guidance in time; (III) reduce the gap: using IOT medical technology to manage PR treatment can reduce the time and space difference of medical knowledge of physicians in Grade 3A hospitals, and also reduce the difference of resources and experience of physicians between three grades of hospitals, accelerating the improvement of rehabilitation treatment level of primary physicians, so that patients can enjoy professional medical health services in their vicinity; (IV) individualize the rehabilitation: individualized PR treatment plans can be provided for different groups of people to fully meet their needs for different levels of rehabilitation treatment, thus meeting the requirements of precision medicine.

Due to the chronic course of COPD and the need for long-term treatment, which requires the active participation and cooperation of patients throughout the whole process (9), the rehabilitation of patients with COPD will become the focus of the application of IOT medicine in the field of rehabilitation of chronic diseases in internal medicine. IOT medical technology played a top-level and pyramidal role of demonstration and influence in the clinical process of chronic disease management and rehabilitation. By establishing a three-tier IOT medical platform that integrates authoritative experts specialized in COPD rehabilitation in large hospitals, primary physicians and patients, forming a multidisciplinary integrated respiratory disease rehabilitation platform, and strengthening close cooperation among physicians in Grade 3A and primary hospitals, and integrating the rehabilitation techniques of traditional medicine with Chinese characteristics, such as sports medicine, Chinese medicine and Tai Chi, into the above multidisciplinary respiratory disease rehabilitation platform, it is possible to engage and guide the implementation of pulmonary rehabilitation for patients with COPD in a comprehensive, holistic, precise and fulltime way.

Applying IOT technology to the management of COPD rehabilitation, realizing the linkage of medical association, two-way referral, remote consultation and home rehabilitation management of COPD, this seamless full management and dynamic data feedback and collection will favorably promote the implementation of COPD rehabilitation training, and its development prospect is broad and potential is unlimited. In addition, China is the most rapidly growing country in the world in terms of Internet development, and the huge volume of Internet users has laid a beneficial foundation for the rehabilitation treatment of COPD in the context of IOT. However, there is a lack of unified understanding and norms for diagnosis and treatment, and the consensus or guidelines for clinical application in the field of IOT pulmonary rehabilitation are even blank. Therefore, we should not limit the application of IOT technology in the rehabilitation of COPD, but also should develop a set of IOT-based rehabilitation treatment model that meets the national condition in China and is suitable for Chinese patients with COPD, for which we invited well-known experts from the field of IOT medicine and COPD pulmonary rehabilitation in China to discuss together in Shanghai to reach this consensus (11).

We present the following article in accordance with the RIGHT reporting checklist (available at https://dx.doi. org/10.21037/jtd-21-670).

\section{IOT-based rehabilitation strategy for COPD}

\section{Centralized management}

The respiratory departments of Grade 3A hospitals are set 
as the first echelon of IOT-based COPD rehabilitation, which mainly provide rehabilitation treatment for difficult COPD patients, provide guidance to the rehabilitation program of general patients, and undertake training for primary health care staffs. The basic information of patients, rehabilitation treatment plans and related data before and after treatment are finally collected by the first echelon, uploaded to the computer cloud for storage, and the collected data are analyzed and studied. Based on the demographic characteristics of China, rehabilitation techniques applicable to Chinese patients with COPD are explored and the IOT rehabilitation model is innovated.

\section{Community rebabilitation treatment}

Community hospitals will serve as the second echelon of IOT-based COPD rehabilitation. Community rehabilitation has always been an important part of rehabilitation medical treatment. Community hospitals mainly undertake the rehabilitation treatment of general COPD patients in their vicinity, instruct patients on formal rehabilitation training movements and monitor their exercise intensity. In addition, community hospitals provide patients with highstandard equipment such as upper and lower extremity muscle training equipment, balance trainers, simulated exercise trainers and hydrotherapy. Rational use of medical resources can reduce the cost of rehabilitation treatment. Community-based rehabilitation can greatly facilitate treatment close to patients, create a mutually supervised environment for patients, improve patient compliance, and also reduce the financial burden on patients. The second echelon of community-based rehabilitation will become an important module in IOT rehabilitation.

\section{Home rehabilitation treatment}

Home rehabilitation is the third echelon of IOT-based COPD rehabilitation, which enables ordinary patients with COPD to change from passive to active rehabilitation treatment at home. The doctor of the first echelon makes a comprehensive assessment of the patient, draws up a rehabilitation program and pushes it to the patient's cell phone APP, which is based on exercise rehabilitation, including aerobic exercise, resistance training, breathing training, and pulmonary rehabilitation exercises. At the same time, through the cell phone APP, patients are educated to improve their health knowledge and selfmanagement. Doctors and rehabilitators can also follow up with patients by voice or video call through the cell phone APP, and make regular remote assessments to ensure real-time tracking of patients' rehabilitation status so that adjustments can be made. In addition, patients can wear wearable devices such as sports bracelets to monitor exercise intensity and physiological indicators (heart rate, blood pressure, etc.). The data collected is uploaded via the Internet to a computer cloud, where it is analyzed by the first echelon, while rehabilitation practitioners in the second echelon assess the patient's exercise performance and adjust the rehabilitation program according to the actual situation.

In the third echelon, patients are able to communicate with their doctors or rehabilitators in a timely manner and provide real-time feedback on their rehabilitation results. Moreover, with the guidance and supervision of cell phones or other wearable devices, patients can complete the corresponding rehabilitation training, reducing their financial burden and improving their compliance. Studies have shown that the economic burden of internetbased tele-rehabilitation was not significantly different from that of traditional rehabilitation, and will decrease with the development of the IOT and the popularity of devices $(9,12,13)$. For patients living in remote areas, telerehabilitation is more economical and practical.

\section{The establishment of a medical working system of IOT for COPD rehabilitation}

\section{Cloud computing devices}

"Cloud" is a virtual computer resource that can be selfmaintained and managed, usually some large server clusters, including computer servers, storage servers, broadband resources and so on (14). The powerful computing and storage capacity of cloud computing can provide strong hardware and software and technical support for the storage and processing of massive data in IOT, which can effectively meet the elastic demand of patient monitoring data processing and analysis of huge computing volume (15). The large amount of monitoring data accumulated can be data mined, which fully reflects the advantages of cloud computing.

The receiving and transmission function of the cloud must be available in the IOT platform software to adapt to the IOT transmission process, transmitting the processed information to the user terminal accurately according to the duty criteria of the terminal and the information access. User terminals can be physicians, rehabilitators and related personnel, patients who need to know information, 


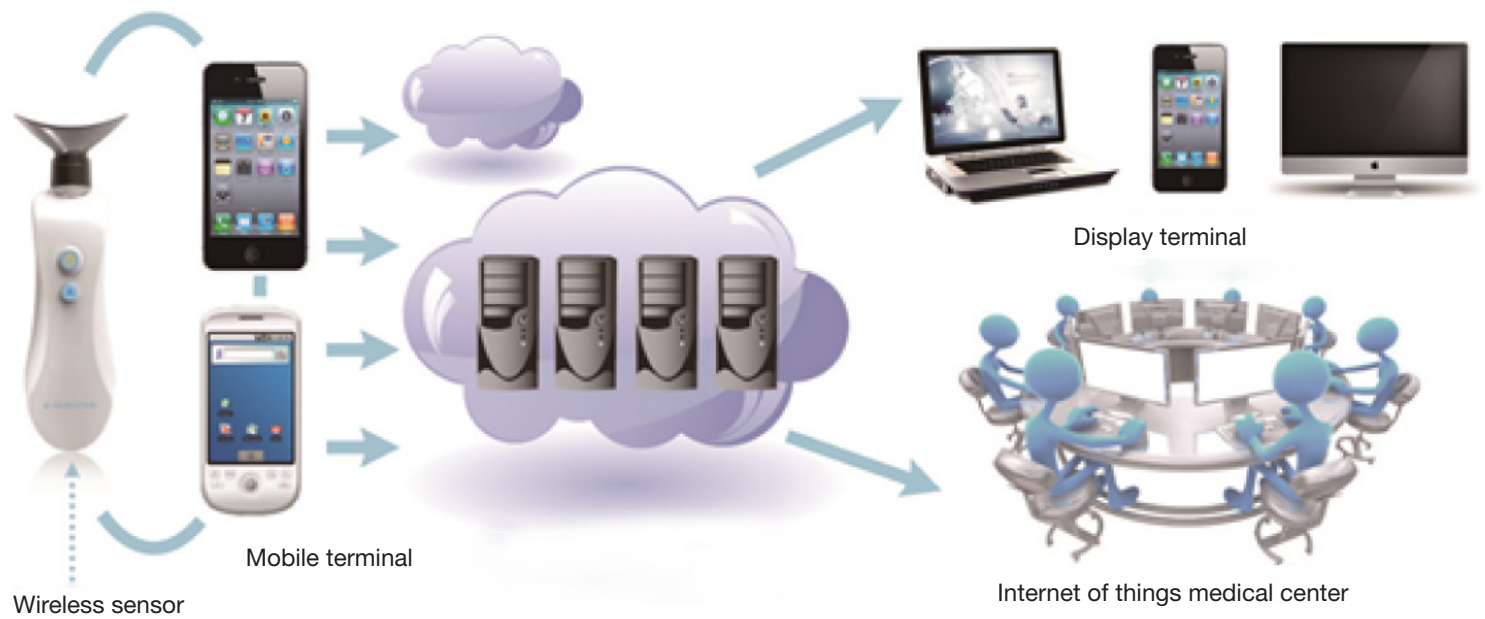

Figure 1 Intelligent massive information mining system based on cloud computing.

informed persons, and administrator terminals. The application of cloud computing technology to the pulmonary rehabilitation IOT system not only enables deep processing and mining of massive amounts of data, but also makes it possible to achieve fast, accurate, high-capacity data that can be collected and processed anytime anywhere (Figure 1).

It connects the patient side, the physician side, the cloud processing side, and also connects with the services of hospitals of three grades, so that it can complete comprehensive treatment services of chronic disease management and rehabilitation as a whole.

\section{Telemedicine system}

Telemedicine monitoring refers to a technical means of transmitting video, physiological information and medical signals from the remote end to physicians and rehabilitators for analysis and dynamic follow-up through communication networks. The telemedicine monitoring system consists of three parts: the physician side, the patient side and the communication network. The function of the physician's side is to receive information from the remote monitoring device and provide the patient with corresponding professional guidance. The remote monitoring device is a device for rehabilitation guidance, physiological parameter detection and telemetry for patients, which can help doctors grasp the patient's condition and provide timely guidance. The physiological parameters detected mainly include: electrocardiogram (ECG), electroencephalography (EEG), heart rate, blood pressure, pulse, respiratory rate, oxyhemoglobin saturation, body temperature, blood sugar, etc. At the same time, the patient with video, virtual reality (VR) and other technologies to achieve remote visual simulation training. Muscle tension, diaphragm movement, energy consumption of flat exercise, changes in vital signs during exercise, changes in energy metabolism, walking distance, questionnaires and other information are collected from the patient side. The physician side do the classification and summary, setting alarm thresholds, which are evaluated by doctors of different levels, to guide the intensity, amplitude, training form, time, etc. The communication network connects the physician's side and the patient's side. Patients can be monitored at home and outdoors. The monitoring process should be real-time. The equipment should be easy to carry and use. The monitoring can be done by patients or doctors. The measurement results can be uploaded to the cloud for storage, analyzed and processed after remote transmission to the physician's side. Remote expert consultation can be available through the network (Figure 2).

The team of Professors Shanqun Li from Zhongshan Hospital, Fudan University, created some Chinese utility model patents with intellectual property rights: "An IOT-based Expiratory Muscle Training System”, "An IOT-based Intelligent Monitoring Wearable System for COPD Rehabilitation Exercise", and "An IOT-based ICF Evaluation System for Respiratory Rehabilitation of COPD", which extend the face-to-face consultation model between physician and patients with COPD for pulmonary rehabilitation, to home management. The concept and technology of overall management and 


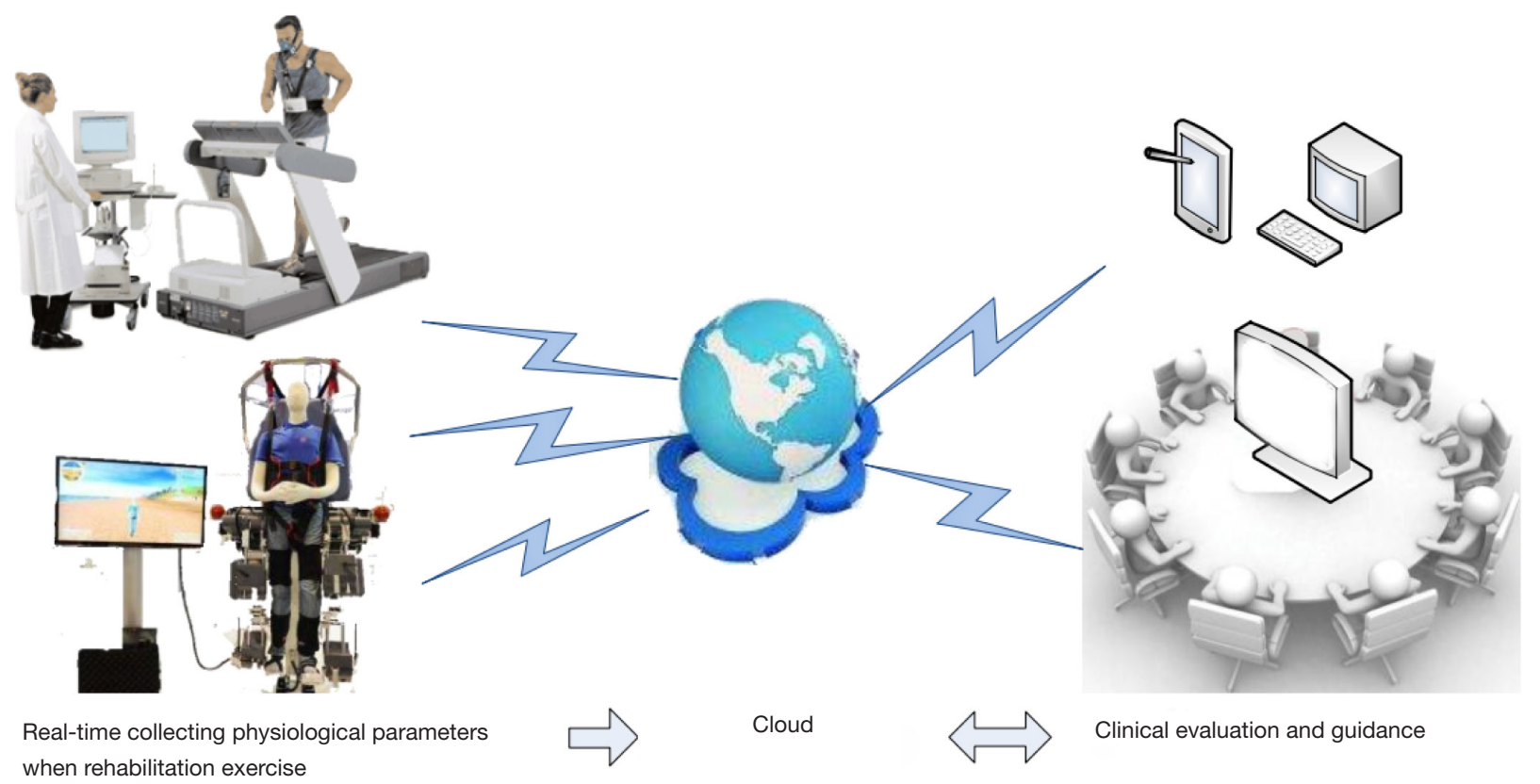

Figure 2 Telemedicine platform for COPD rehabilitation. COPD, chronic obstructive pulmonary disease.

full-time rehabilitation is extended to the family and community.

\section{Implementation criteria for COPD rehabilitation}

\section{Exercise program}

Exercise training is the cornerstone of comprehensive PR program, mainly including: aerobic exercise, upper and lower limb resistance training, respiratory training, respiratory muscle training, functional training, etc. (16).

\section{Exercise training intensity}

The American College of Sports Medicine (ACSM) and American Association of Cardiovascular and Pulmonary Rehabilitation (AACVPR) recommended low-intensity (30-40\% maximal power) and high-intensity (60-80\% maximal power) exercise training for patients with COPD (17). Low-intensity can relieve symptoms, improve quality of life, and enhance physical activity in daily life, while higher-intensity exercise can lead to significant improvement in physiological function. The indicators of exercise intensity assessment include: maximum oxygen uptake (VO2max), oxygen uptake reserve (VO2R), metabolic equivalent, maximum heart rate (HRmax), heart rate reserve (HRR), subjective physical sensation scale (ratings of perceived exertion, RPE score), Borg dyspnea score (Borg score), etc.

\section{Rebabilitation assessment}

One-min sitting test, 6-min walking distance, simple lung function, cardiopulmonary exercise test, etc. to detect lung function. Questionnaires to assess anxiety and depressive symptoms in patients with COPD, including: BORG (B for body mass index, $\mathrm{O}$ for airflow obstruction, $\mathrm{D}$ for dyspnea, E for exercise capacity) and MRC (Medical Research Council dyspnea scale) to assess the degree of dyspnea, CAT (COPD Assessment Test) questionnaire to measure quality of life, Hamilton Anxiety Rating Scale (HARS) and Hamilton Depression Rating Scale (HDRS) to quantify anxiety and depression as a routine screening procedure to detect early symptoms and halt their progression.

\section{Long-term oxygen therapy and non-invasive ventilation}

In patients with COPD even in the absence of hypoxemia, small increase in oxygenation index can lead to significant improvements in exercise tolerance, rising to approximately $50 \%$ of the oxygenation index level (18). Reduction of respiratory muscle power consumption, relief of muscle 
fatigue and good man-machine synchronization are the main objectives of noninvasive ventilation.

Non-invasive ventilation modes commonly used in exercise training for patients with COPD include: biphasic positive airway pressure (BiPAP), proportional assisted ventilation (PAV), and adaptive support ventilation (ASV), pressure support ventilation (PSV), and so on. The most commonly used ventilation mode is BiPAP. Studies have shown that PSV mode has the advantage of reducing the work done by respiratory muscles, while PAV mode has the main advantage of better human-machine synchronization (19). Studies have confirmed that the use of IOT technology for rehabilitation training, including remote home noninvasive ventilation management, can improve the quality of noninvasive ventilation, improve treatment compliance, and contribute to the improvement of patient survival (10).

\section{Other interventions}

\section{Health education}

Health education is infiltrated into all aspects of PR, which can improve the patient's knowledge and confidence in long-term treatment, and reduce the number of acute attacks. The health education content will be pushed to the patients' cell phone APP by the physicians and rehabilitation teachers in the form of graphics or videos, including: risk factors of COPD, intervention measures, methods of medication, hazards of smoking, methods of PR treatment, self-monitoring, self-management of symptoms, importance of adherence to pulmonary rehabilitation, etc.

\section{Nutritional therapy}

Nutritional therapy is part of the individualized treatment plan, especially for patients with COPD who have combined diabetes, metabolic syndrome and malnutrition. The diet is based on the intake of protein, and the fat and carbohydrate content can be relatively low, thus reducing the production of carbon dioxide. In patients with COPD, fat-free weight loss is considered a poor prognostic parameter that will lead to muscle weakness and reduced quality of life (20). Fat weight loss can be assessed using bioelectrical impedance analysis. Individual nutritional therapy is an effective and underestimated intervention for the treatment of COPD, especially in malnourished patients and during physical activity.

\section{Assessment of psychological status}

Patients with COPD have long disease duration, different degrees of exercise endurance and muscle strength incoordination, etc., which all aggravate the psychological burden of patients, so that patients generally have negative emotions such as anxiety and depression. Therefore, doctors and rehabilitation physicians need to provide early and positive psychological interventions to enhance the rehabilitation effect of patients.

\section{Social support}

It was found that rehabilitation partners were more conducive to patients to establish the belief of completing the task (21). The researchers believe that rehabilitation partners participating in training with patients with COPD, can give explanation, relief and guidance when patients have adverse physiological reactions (soreness and weakness, difficulty in breathing, etc.) during home pulmonary rehabilitation, so that patients can obtain care and supports from their families, which creates a good social and psychological environment for patients and achieves a continuous positive reinforcement of the behavioral intervention process.

\section{Basic requirements of training and admission of professionals}

\section{Training of professionals}

The main training includes: (I) training in the knowledge and diagnosis and treatment techniques of COPD and related diseases; (II) training in the basic knowledge of pulmonary rehabilitation; (III) medical exercise therapy, posture training, muscle function training, pulmonary endurance training; (IV) physical examination; (V) training in the basic knowledge of IOT medical technology; (VI) IOT-based pulmonary rehabilitation working model and process, and roles and responsibilities of each member in a multi-team working model; (VII) application of medical knowledge and IOT technology in the long-term management of patients.

\section{Admission of professionals}

Relevant practitioners need to undergo the necessary 
training, have the relevant medical professional qualifications and obtain a certificate of competence before access. The access and quality control requirements of the IOT COPD Rehabilitation Center are as follows: (I) the center has relevant practitioners (including several full-time rehabilitators who have obtained professional training certificates); (II) the center possesses equipment for conducting relevant pulmonary rehabilitation; (III) the center has certification and access to the IOT COPD rehabilitation; (IV) the center establishes a standardized system for monitoring COPD diagnosis and treatment, rehabilitation training, and exercise intensity; (V) the center has the ability to manage and ensure the security of information.

\section{Conclusions}

In the process of standardized COPD rehabilitation management, the use of modern IOT technology to comprehensively assess patients' physiological and psychological status, and integrating medication, rehabilitation training, psychological intervention and prevention, make the rehabilitation training become an effective supplement to daily clinical work and a way to promote disease recovery. At the same time, it is a new tool to cultivate professional talents, promote discipline development, and drive the transformation of industryacademia-research, which is in line with the current medical status and national policies. The construction of IOT-based three-stage rehabilitation for COPD echoes the three-stage rehabilitation model proposed by China. As people become more aware of rehabilitation, three-stage rehabilitation will become more and more superior. The "cloud hospital" in mobile health care uses cloud computing, IOT, mobile Internet and sensor technology to combine chronic disease management and rehabilitation training, which can significantly improve the data management ability and improve the quality of rehabilitation training, achieving effective dynamic assessment, positive feedback, and also improve patient compliance. The massive data analysis and mining of IOT provides an effective tool to reveal new patterns of disease change.

\section{Acknowledgments}

Funding: The National Key Research and Development Program of China (No. 2018YFC1313600), the National Natural Science Foundation of China (No. 82070094), the Technical Standard Project of Shanghai Science and Technology Innovation Action Plan (No. 20DZ2202300), the Major Project Matching Funding Project of Shanghai Science and Technology Commission (No. 198012309), the Shanghai Key Laboratory of Traditional Chinese Clinical Medicine (No. 20DZ2272200), and the Shanghai Key Clinical Specialty (No. SHSLCZDZK05101).

\section{Footnote}

Reporting Checklist: The authors have completed the RIGHT reporting checklist. Available at https://dx.doi. org/10.21037/jtd-21-670

Conflicts of Interest: All authors have completed the ICMJE uniform disclosure form (available at https://dx.doi. org/10.21037/jtd-21-670). The authors have no conflicts of interest to declare.

Ethical Statement: The authors are accountable for all aspects of the work in ensuring that questions related to the accuracy or integrity of any part of the work are appropriately investigated and resolved.

Open Access Statement: This is an Open Access article distributed in accordance with the Creative Commons Attribution-NonCommercial-NoDerivs 4.0 International License (CC BY-NC-ND 4.0), which permits the noncommercial replication and distribution of the article with the strict proviso that no changes or edits are made and the original work is properly cited (including links to both the formal publication through the relevant DOI and the license). See: https://creativecommons.org/licenses/by-nc-nd/4.0/.

\section{References}

1. Wang C, Xu J, Yang L, et al. Prevalence and risk factors of chronic obstructive pulmonary disease in China (the China Pulmonary Health CPH study): a national cross-sectional study. Lancet 2018;391:1706-17.

2. Chan KY, Li X, Chen W, et al. Prevalence of chronic obstructive pulmonary disease (COPD) in China in 1990 and 2010. J Glob Health 2017;7:020704.

3. Spruit MA, Singh SJ, Garvey C, et al. An official American Thoracic Society/European Respiratory Society statement: key concepts and advances in pulmonary rehabilitation. Am J Respir Crit Care Med 2013;188:e13-64.

4. Wedzicha JA Ers Co-Chair, Miravitlles M, Hurst JR, et 
al. Management of COPD exacerbations: a European Respiratory Society/American Thoracic Society guideline. Eur Respir J 2017;49:1600791.

5. Singh D, Agusti A, Anzueto A, et al. Global Strategy for the Diagnosis, Management, and Prevention of Chronic Obstructive Lung Disease: the GOLD science committee report 2019. Eur Respir J 2019;53:1900164.

6. Halpin DMG, Criner GJ, Papi A, et al. Global Initiative for the Diagnosis, Management, and Prevention of Chronic Obstructive Lung Disease. The 2020 GOLD Science Committee Report on COVID-19 and Chronic Obstructive Pulmonary Disease. Am J Respir Crit Care Med 2021;203:24-36.

7. Kelly JT, Campbell KL, Gong E, et al. The Internet of Things: Impact and Implications for Health Care Delivery. J Med Internet Res 2020;22:e20135.

8. Bai L, Yang D, Wang X, et al. Chinese experts' consensus on the Internet of Things-aided diagnosis and treatment of coronavirus disease 2019 (COVID-19). Clinical eHealth 2020;3:7-15.

9. Zhang J, Song YL, Bai CX. MIOTIC study: a prospective, multicenter, randomized study to evaluate the long-term efficacy of mobile phone-based Internet of Things in the management of patients with stable COPD. Int J Chron Obstruct Pulmon Dis 2013;8:433-8.

10. Jiang W, Wang L, Song Y. Titration and follow-up for home noninvasive positive pressure ventilation in chronic obstructive pulmonary disease: The potential role of tele-monitoring and the Internet of things. Clin Respir J 2021;15:705-15.

11. Lao X, Zhang J, Bai C. The implication of telehealthcare in COPD management of China. Expert Rev Respir Med 2013;7:459-63.

12. Nelson M, Russell T, Crossley K, et al. Cost-effectiveness

Cite this article as: Xiang $\mathrm{G}, \mathrm{Zhu} \mathrm{X}, \mathrm{Ma}$ L, Huang H, Wu X, Zhang W, Li S; on behalf of the Writing Expert Group of Expert Consensus on Clinical Application of IOT Medical Technology in the Rehabilitation of Chronic Obstructive Pulmonary Disease, the Respiratory Disease Rehabilitation Professional Committee of China Medical Education Association. Clinical guidelines on the application of Internet of Things (IOT) medical technology in the rehabilitation of chronic obstructive pulmonary disease. J Thorac Dis 2021;13(8):4629-4637. doi: 10.21037/jtd-21-670 of telerehabilitation versus traditional care after total hip replacement: A trial-based economic evaluation. J Telemed Telecare 2021;27:359-66.

13. Fatoye F, Gebrye T, Fatoye C, et al. The Clinical and Cost-Effectiveness of Telerehabilitation for People With Nonspecific Chronic Low Back Pain: Randomized Controlled Trial. JMIR Mhealth Uhealth 2020;8:e15375.

14. Weber N, Liou D, Dommer J, et al. Nephele: a cloud platform for simplified, standardized and reproducible microbiome data analysis. Bioinformatics 2018;34:1411-3.

15. Nadian-Ghomsheh A, Farahani B, Kavian M. A hierarchical privacy-preserving Io $\mathrm{T}$ architecture for visionbased hand rehabilitation assessment. Multimed Tools Appl 2021:1-24.

16. Nolan CM, Rochester CL. Exercise Training Modalities for People with Chronic Obstructive Pulmonary Disease. COPD 2019;16:378-89.

17. Bennett SB, Pescatello LS. A regional comparison of cardiac rehabilitation personnel. Adherence to the 1995 American Association of Cardiovascular and Pulmonary Rehabilitation Guidelines by Staff Position. J Cardiopulm Rehabil 1997;17:92-102.

18. Vitacca M, Paneroni M, Zampogna E, et al. HighFlow Oxygen Therapy During Exercise Training in Patients With Chronic Obstructive Pulmonary Disease and Chronic Hypoxemia: A Multicenter Randomized Controlled Trial. Phys Ther 2020;100:1249-59.

19. Ambrosino N, Xie L. The Use of Non-invasive Ventilation during Exercise Training in COPD Patients. COPD 2017;14:396-400.

20. Keogh E, Mark Williams E. Managing malnutrition in COPD: A review. Respir Med 2021;176:106248.

21. Oh-Park M, Lew HL, Raghavan P. Telerehabilitation for Geriatrics. Phys Med Rehabil Clin N Am 2021;32:291-305. 